\title{
Araceae endémicas del Perú
}

Jorge Lingán

Museo de Historia Natural, Av. Arenales 1256, Aptdo. 14-0434, Lima 14, Perú. jorge lingan@hotmail.com

\section{Resumen}

La familia Araceae es reconocida en el Perú con 26 géneros y alrededor de 250 especies (Brako \& Zarucchi, 1993; Ulloa Ulloa et al., 2004), mayormente hierbas epífitas o hemiepífitas. En este trabajo reconocemos 52 endemismos, en nueve géneros. Uno de estos géneros, Filarum, es endémico. Estos taxones endémicos ocupan los Bosques Pluvial Montanos y Húmedos Montanos, así como la región del Bosque Húmedo Amazónico, entre los 100 y 3000 m de altitud. Trece de estas especies se encuentran representadas en áreas naturales protegidas.

Palabras claves: Araceae, Perú, endemismo, plantas endémicas.

\section{Abstract}

The Araceae are represented in Peru with 26 genera and nearly 250 species (Brako \& Zarucchi, 1993; Ulloa Ulloa et al., 2004), most of them herbaceous epiphytes or hemiepiphytes. Here we recognize 52 endemic taxa in nine genera. One genus, Filarum, is endemic to Peru. Endemic aroids are found in Pluvial and Humid Montane Forests, and in the Humid Amazonian Forest, between 100 and 3000 m elevation. Thirteen endemic species have been registered within Peru's protected areas network.

Keywords: Araceae, Filarum, aroids, Peru, endemism, endemic species.

\section{Anthunium cainarachense Engl.}

\section{CR, Blab(iii)}

Publicación: Pflanzenr. IV. 23B (Heft 21): 192. 1905. Colección tipo: E.H.G. Ule 6326

Herbarios: B.

Nombre común: D esconocido.

Registro departamental: SM.

Regiones Ecológicas: BHA; altitud desconocida.

SINANPE: Sin registro.

Herbarios peruanos: Ninguno.

Observaciones: Planta escandente con espádice rojizo, conocida solamente de una localidad, en San Martín, en la cuenca del río Cainarache, afluente del Huallaga. No ha vuelto a ser recolectada desde 1902. Probablemente se trate de una especie de ambientes boscosos, que en esa localidad están afectados por la deforestación.

\section{Anthurium carneospadix Engl.}

\section{DD}

Publicación: Pflanzenr. IV 23B(Heft21): 194. 1903.

Colección tipo: A. Weberbauer 2115

Herbarios: B.

Nombre común: D esconocido.

Registro departamental: CA, JU.

Regiones Ecológicas: BMHM; 2000- $2700 \mathrm{~m}$.

SINANPE: Sin registro.

Herbarios peruanos: Ninguno.

Observaciones: Especie herbácea conocida sólo de unas pocas localidades, separadas entre ellas por más de $600 \mathrm{~km}$. No fue listada como endémica para el Perú (Brako \& Zarucchi, 1993). Macbride (1936) incluyó un ejemplar de Weberbauer, recolectado en la provincia de Hualgayoc, que representaría a esta especie en la vertiente occidental, pero no ha vuelto a ser registrada ahí, desde 1904. No se cuenta con información adicional sobre su hábitat y situación de sus poblaciones.

\section{Anthurium chinchipense Croat \& Lingán}

$$
\text { EN, Bla }
$$

Publicación: Rodriguesia 56(88): 43-44, f. 1a. 2005.

Colección tipo: J. Campos et al. 6200

Herbarios: B, K, MO, NY, US; USM!.

Nombre común: D esconocido.

Registro departamental: CA.

Regiones Ecológicas: BMHP; $900 \mathrm{~m}$.

SINANPE: Sin registro.

Henbarios penuanos: USM (isotipo+1).

Observaciones: Especie herbácea, descrita y conocida de la cuenca del Chinchipe en el norte del país. Amenazas a sus poblaciones podrían estar asociadas a la deforestación.

\section{Anthurium consimile Schott}

\section{VU, B lab(iii)}

Publicación: O esterr. Bot. Wochenbl. 7: 294. 1857.

Colección tipo: E.F. Poeppig 1083

Herbarios: P, W.

Nombre común: D esconocido.

Registro departamental: HU, JU, PA, SM.

Regiones Ecológicas: BMHP; 500-1100 m.

SINAN PE: Sin registro.

Herbarios penuanos: USM (7).

Observaciones: Especie herbácea que no fue señalada como endémica en Brako \& Zarucchi (1993). Se conoce del norte y centro del país, siempre en ambientes boscosos. Cuenta con recolectas recientes, aunque amenazas a sus poblaciones provienen de la deforestación asociada a la agricultura. Ha sido recolectada cerca al Parque Nacional Yanachaga-Chemillén.

Nota del Editor: En la versión on line de este artículo han sido omitidos los mapas del Perú que ilustraban el Registro departamental. Para ubicar las abreviaturas de los departamentos vea al final del artículo. 


\section{Anthurium gracilipedunculatum K. Krause}

$$
\text { EN, Blab(iii) }
$$

Publicación: Publ. Field Columbian Mus. Bot. Ser. 8(2): 78. 1930.

Colección tipo: J.F. Macbride 3734

Herbarios: F, US

Nombre común: D esconocido.

Registro departamental: CA, HU, PA.

Regiones Ecológicas: BMHM; 1500$2600 \mathrm{~m}$.

SINANPE: PNC, PNYC

Herbanios penuanos: AMAZ (1), HUT (1), MOL (1), USM (4).

Observaciones: Especie herbácea conocida de varias localidades, en la vertiente oriental andina. Las localidades más distantes entre ellas están ubicadas aproximadamente $500 \mathrm{~km}$ aparte. Ejemplares de herbario recolectados, entre 1990 y 2003, provienen de dos áreas protegidas. Probablemente, la creciente deforestación y/ 0 fragmentación de bosques constituyen amenazas a las poblaciones fuera de las áreas protegidas.

\section{Anthurium hamiltonii Croat \& Lingán}

\section{VU, Bla}

Publicación: Rodriguesia 56(88): 44, 4647, f. 2. 2005.

Colección tipo: J. Lingán et al. 342

Herbarios: MO.

Nombre común: D esconocido.

Registro departamental: CA, PA.

Regiones Ecológicas: BMHM; 2200$2500 \mathrm{~m}$.

SINANPE: PNC, PNYC

Hembarios peruanos: Ninguno.

Observaciones: Especie herbácea, conocida de poblaciones, al parecer, disyuntas por más de $600 \mathrm{~km}$ en línea recta. Las dos poblaciones conocidas provienen de áreas protegidas, fuera de las cuales, amenazas a sus poblaciones provienen de la deforestación.

\section{Anthunium huallagense Engl.}

\section{VU, B lab(iii)}

Publicación: Pflanzenr. 23 B (Heft 23): 132. 1905.

Colección tipo: A. Weberbauer 3660

Herbarios: B.

Nombre común: D esconocido.

Registro departamental: HU, JU, PA. Regiones Ecológicas: BMHM, BMHP; 450- $1700 \mathrm{~m}$.

SINANPE: PNYC

Herbarios peruanos: USM (2).

Observaciones: Especie epífita conocida del centro del país, presente en un área aproximada de $8000 \mathrm{~km}^{2}$. Solamente una subpoblación está registrada en un área protegida. Amenazas a sus poblaciones provienen, probablemente, de la deforestación por ampliación de la actividad agrícola.

\section{Anthunium huanucense Engl.}

$$
\text { EN, Blab(iii) }
$$

Publicación: Pflanzenr. IV, 23B (Heft 21): 165. 1905.

Colección tipo: A. Weberbauer 3449

Herbarios: $B$.

Nombre común: D esconocido.

Registro departamental: HU, JU.

Regiones Ecológicas: BMHP; 900- $1500 \mathrm{~m}$.

SINANPE: Sin registro.

Herbarios peruanos: Ninguno.

Observaciones: Especie herbácea conocida solamente de tres localidades, en el centro del país: Una en la cuenca del Monzón y las otras dos en las del Huallagay Tulumayo. Las localidades más alejadas se hallan separadas por aproximadamente $300 \mathrm{~km}$. Probablemente, la deforestación sea la amenaza principal al hábitat de esta especie.

\section{Anthunium idmense K. Krause}

$$
\text { EN, Blab(iii) }
$$

Publicación: Notizbl. Bot. Gart. BerlinDahlem 6: 608-609. 1932.

Colección tipo: A. Weberbauer 5036

Hemarios: $B$.

Nombre común: D esconocido.

Registro departamental: CA, CU.

Regiones Ecológicas: BMHM; 1700$2200 \mathrm{~m}$

SINANPE: Sin registro.

Hemarios penuanos: HAO (1).

Observaciones: Especie epífita conocida de dos localidades aisladas, separadas entre ellas por más de $1000 \mathrm{~km}$ de distancia. Fue descrita de una planta recolectada en la cuenca del Unubamba, en 1905. Habita los bosques montanos y la deforestación es una amenza a sus poblaciones.

\section{Anthunium knappiae Croat}

$$
\text { EN, Blab(iii) }
$$

Publicación: Ann. Missouri Bot. Gard. 78(3): 665-666. 1991.

Colección tipo: S. Knapp et al. 8544

Hembarios: B, MO, US; USM!.

Nombre común: Vaca mansana.

Registro departamental: SM.

Regiones Ecológicas: BHA; 400-500 m. SINANPE: Sin registro.

Herbarios peruanos: USM (isotipo).

Observaciones: Especie epífita conocida, al parecer, de una localidad, en la cuenca del río Huallaga. Esta localidad, cerca de Shapajilla, ha sido explorada durante el siglo XIX e inicios del XX; sin embargo, cerros aislados y valles cerrados encierran una flora particular que podría incluir a esta especie.

\section{Anthurium latissimum Engl.}

\section{VU, B lab(iii)}

Publicación: Pflanzenr. IV, 23B (Heft 21): 292. 1905.

Colección tipo: A. Weberbauer 1939

Henbarios: B, F, MO.

Nombre común: D esconocido. Registro departamental: AY, HU, JU, PA, SM.

Regiones Ecológicas: BMHP; 500— 2000 m. SINAN PE: PNYC

Herbarios peruanos: USM (4). 
Observaciones: Especie herbácea conocida de casi 10 localidades, en el oriente del país. La distancia entre las localidades extremas es de aproximadamente $900 \mathrm{~km}$, con las poblaciones ocupando valles aislados, como el del Apurímac y del Huallaga. Ha sido recolectada en varias oportunidades de bosques secundarios.

\section{Anthurium llewelynii Croat}

\section{VU, Blab(iii)}

Publicación: Ann. Missouri Bot. Gard. 78(3): 674, f. 181, 183-185. 1991.

Colección tipo: T. Croat 51092

Hemarios: AAU, B, CN, DUKE, ENCB, IBE, JBGP, K, KYO, L, LE, M, MO, NY, RSA, SAR, SEL, TEX, US; USM!.

Nombre común: D esconocido.

Registro departamental: HU, SM.

Regiones Ecológicas: $\mathrm{BMHP}, \mathrm{BHA}$; 130- $900 \mathrm{~m}$.

SINAN PE: Sin registro.

Hemarios peruanos: USM (isotipo).

Observaciones: Esta hierba terrestre se conoce de las cuencas de los ríos Huallaga y Mayo, ocupando bosques con estacionalidad de lluvias marcada. Ha sido registrada en suelos arenosos, por lo que es posible se trata de una especialista de hábitat.

\section{Anthurium macbridei K. Krause}

$$
\text { CR, Blab(iii) }
$$

Publicación: Publ. Field Columbian Mus., Bot. Ser. 8(2): 79. 1930

Colección tipo: J.F. Macbride 4110

Herbarios: $F$.

Nombre común: D esconocido.

Registro departamental: HU.

Regiones Ecológicas: BMHM; $1950 \mathrm{~m}$.

SINAN PE: Sin registro.

Herbarios peruanos: Ninguno.

Observaciones: Esta especie herbácea se conoce de una localidad, en la cuenca del Monzón, la cual está afectada por la deforestación y cambios en su paisaje por actividad humana. Aparentemente no ha vuelto a ser recolectada desde 1923.

\section{Anthunium magdaeCroat \& Lingán}

$$
\text { VU, Bla }
$$

Publicación: Rodriguesia 56(88): 47-48, f. 3a. 2005 .

Colección tipo: C. Díaz \& A. Torres 7832

Hemarios: MO; USM!.

Nombre común: D esconocido.

Registro departamental: AM, CA, JU.

Regiones Ecológicas: BMHM, BMHP; $1400-2300 \mathrm{~m}$.

SINANPE: Sin registro.

Herbarios peruanos: USM (1).

Observaciones: Especie herbácea, terrestre o epífita baja, descrita de una colección de 1995, proveniente de la cuenca del Chinchipe. Se conoce de otras poblaciones dispersas, como en la cuenca del Tulumayo.

\section{Anthurium manuanum Croat}

EN, Bla

Publicación: Ann. Missouri Bot. Gard. 78(3): 680, f. 192A. 1991.

Colección tipo: C. Vargas C. 17747

Hembarios: CUZ!

Nombre común: D esconocido.

Registro departamental: MD.

Regiones Ecológicas: $\mathrm{BMHP}, \mathrm{BHA}$; 260-840 m.

SINANPE: PNM

Hemarios peruanos: CUZ (isotipo).

Observaciones: Esta especie epífita se conoce solamente de las cuencas de los ríos Alto Madre de Dios y Madre de Dios. Si bien su distribución es restringida, sus poblaciones se hallan en las cercanías 0 en el interior de un área protegida. Se la reconoce aquí como amenazada, por su área de presencia limitada.

\section{Anthurium mariae Croat \& Lingán}

$$
\text { CR, B lab(iii) }
$$

Publicación: Rodriguesia 56(88): 49-50, f. 3b. 2005.

Colección tipo: K.R. Young \& M. Eisenberg 314

Hembarios: B, K, MO.

Nombre común: D esconocido.

Registro departamental: AM.

Regiones Ecológicas: BMHP; 1300 m.

SINAN PE: Sin registro.

Herbarios peruanos: Ninguno.

Observaciones: Hierba epífita conocida, aparentemente, sólo de la localidad tipo, en la cuenca del Utcubamba, en un fragmento de bosque montano bajo; esa localidad está severamente afectada por la expansión de la agricultura y colonización.

\section{Anthurium monzonense Engl.}

\section{VU, Blab(iv)}

Publicación: Pflanzenr. IV, 23B (Heft 21): 264. 1905.

Colección tipo: A. Weberbauer 3562

Herbarios: B.

Nombre común: D esconocido.

Registro departamental: CU, HU, JU, PA, SM.

Regiones Ecológicas: BMHM; 1400 $1900 \mathrm{~m}$.

SINAN PE: Sin registro.

Herbarios penuanos: USM (1).

Observaciones: Esta hierba se conoce de localidades naturalmente fragmentadas, en cima de cordilleras aisladas. Fue descrita de una planta recolectada en 1903, de la cuenca del Monzón, la cual además de incluir varios endemismos, está extensamente modificada por ampliación de la frontera agrícola. 
18. Anthurium peltatum Poepp.

$$
\text { VU, B lab(iii) }
$$

Publicación: Nov. Gen. Sp. Pl. 3: 83. 1845. Colección tipo: E.F. Poeppig s.n.

Herbarios: B.

Nombre común: Desconocido.

Registro departamental: AM, HU, SM, UC. Regiones Ecológicas: BMHM, BMHP; $400-1380 \mathrm{~m}$.

SINAN PE: Sin registro.

Herbarios peruanos: USM (3).

Observaciones: Esta hierba se conoce de más de cinco localidades, en las cuencas del Huallaga, Chinchipe y Ucayali. En el tratamiento de Macbride (1936), así como en Brako \& Zarucchi (1993) el nombre de estaespeciefueconsiderado como $\mathrm{H}$ omalomena petata. Hasido recolectada en los límites sur del Parque Nacional Cordillera Azul.

\section{Anthurium reflexinervium Croat}

\section{EN, Blab(iv)}

Publicación: Ann. Missouri Bot. Gard. 78(3): 713, f. 267, 268, 271. 1991.

Colección tipo: T.C. Plowman \& $\mathrm{M}$. Ramirez 7585

Herbarios: F, K, MO, NY, SEL; USM!.

Nombre común: D esconocido.

Registro departamental: HU.

Regiones Ecológicas: BMHP; 650- 800 m.

SINAN PE: PNTM

Herbarios peruanos: USM (isotipo+3).

Observaciones: Esta hermosa hierba se conoce de poblaciones muy pequeñas, ubicadas en los alrededores deTingo María. Se conoce también de plantas cultivadas como ornamentales, en jardínes botánicos y privados. Al parecer, podría tratarse de una endémica con especificidad de substrato a rocas calcáreas.

\section{Anthunium remotigeniculatum Croat}

\section{VU, B lab(iii)}

Publicación: Ann. Missouri Bot. Gard. 78(3): 714-715, f. 272, 275. 1991.

Colección tipo: T. Croat 58114

Herbarios: B, GH, K, M, MO, NY, QCA, RSA, SEL, TEX, US.

Nombre común: D esconocido.

Registro departamental: AM, SM.

Regiones Ecológicas: BMHP; 530 - $1160 \mathrm{~m}$.

SINAN PE: Sin registro.

Hemarios peruanos: Ninguno.

Observaciones: Esta especie herbácea no fue reconocida en Brako \& Zarucchi (1993) como endémica. Ha sido registrada en las cuencas de losníos Marañón yHuallaga, donde ocupaladeras rocosas pronunciadas.

\section{Anthurium schunkei K. Krause}

\section{CR, B 1ab(iii)}

Publicación: Publ. Field Columbian Mus. Bot. Ser. 8(2): 77. 1930.

Colección tipo: C.O. Schunke 293

Herbarios: F.

Nombre común: D esconocido.

Registro departamental: JU.

Regiones Ecológicas: BMHP; $1200 \mathrm{~m}$.

SINANPE: Sin registro.

Herbarios peruanos: Ninguno.
Observaciones: Esta especie herbácea se conoce sólo de una localidad, ubicada en la cuenca del río Chanchamayo. No ha vuelto a ser recolectada desde mediados de la década de 1920, aún cuando este valle ha recibido herborización continua. Por su distribución tan limitada y la falta de representación en el sistema de áreas protegidas, se la considera amenazada.

\section{Anthunium simpsonii Croat}

$$
\text { CR, Blab(iii) }
$$

Publicación: Ann. Missouri Bot. Gard. 78(3): 728, f. 290. 1991.

Colección tipo: D.R. Simpson \& J. Schunke V. 392

Herbarios: F; USM!.

Nombre común: D esconocido.

Registro departamental: TU.

Regiones Ecológicas: BS; $600-800 \mathrm{~m}$.

SINAN PE: ZRT

Herbarios peruanos: USM (isotipo).

Observaciones: Hierba epífita, rara, conocida de una localidad en la Zona Reservada de Tumbes. Aparentemente, no ha vuelto a ser recolectada desde 1967. Podría estar representada en la flora ecuatoriana.

\section{Anthurium superbum Madison subsp. brentbenlinii Croat EN, Blab(iii)}

Publicación: Ann. Missouri Bot. Gard. 78(3): 737, f. 310. 1991

Colección tipo: B. Berlin 644

Herbarios: MO, NY.

Nombre común: Desconocido.

Registro departamental: AM.

Regiones Ecológicas: BHA; 180-350 m. SINAN PE: ZRSC

Herbarios peruanos: Ninguno.

Observaciones: Hierba epífita, conocida de la Zona Reservada Santiago-Comaina, en la cuenca del río Cenepa. Todas las colecciones de herbario fueron realizadas en la década de 1970 y no ha vuelto, al parecer, a ser recolectada.

\section{Anthurium tarapotense Engl.}

\section{VU, B lab(iii)}

Publicación: Pflanzenr. IV, 23B (Heft 21): 74. 1905.

Colección tipo: E.H.G. Ule 5p

Hemarios: B.

Nombre común: D esconocido.

Registro departamental: PA, SM.

Regiones Ecológicas: $\mathrm{BMHP}, \mathrm{BHA}$; $300-1320 \mathrm{~m}$.

SINAN PE: Sin registro.

Herbarios peruanos: USM (1).

Observaciones: Esta hierba se conoce de más de cinco localidades, con remanentes de bosque premontano, en las cuencas de los ríos Tocache, Huallaga, Mayo y Cacazú. Presenta un rango altitudinal de $1000 \mathrm{~m}$, habitando bosques intervenidos. 
25. Anthurium uleanum Engl. var. nanayense Croat

$$
\text { EN, Blab(iv) }
$$

Publicación: Ann. Missouri Bot. Gard. 78(3): 742, f. 318. 1991.

Colección tipo: J. Solomon 3562

Hemarios: MO.

Nombre común: Kachíniak, Patquina.

Registro departamental: LO.

Regiones Ecológicas: BHA; 100- $160 \mathrm{~m}$.

SINAN PE: RNAM

Herbarios peruanos: USM (2).

Observaciones: Hierba epífita, conocida de cinco localidades, de las cuencas del Momón, Nanay, Tepichey Yaguasyacu. Una población de este taxón está ubicada en la Reserva Nacional Allpahuayo Mishana.

\section{Anthurium yunimaguense Engl. ex K. Krause}

\section{DD}

Publicación: Notizbl. Bot. G art. BerlinDahlem 11: 613. 1932.

Colección tipo: E.H.G. Ule 6307

Herbarios: B.

Nombre común: D esconocido.

Registro departamental: LO.

Regiones Ecológicas: BHA; altitud desconocida.

SINANPE: Sin registro.

Herbarios peruanos: Ninguno.

Observaciones: Bejuco conocido de una localidad, en la cuenca del Huallaga, en el occidente de Loreto. Poco se sabe de su ecología y de sus poblaciones. No ha vuelto a ser recolectada desde 1902.

\section{Dieffenbachia cordata Engl.}

$$
\text { CR, Blab(iii) }
$$

Publicación: Bot. Jahrb. Syst. 37: 135. 1905. Colección tipo: A. Weberbauer 1807 Hembarios: B.

Nombre común: D esconocido.

Registro departamental: JU.

Regiones Ecológicas: BMHP; $1000 \mathrm{~m}$.

SINAN PE: Sin registro.

Herbarios peruanos: Ninguno.

Observaciones: Esta hierba fue descrita de un ejemplar procedente del valle del Chanchamayo, cuenca del Tulumayo, la cual presenta sus ambientes naturales impactados por la colonización creciente. Aparentemente no ha vuelto a ser recolectada desde 1902.

\section{Dieffenbachia imperialis Linden \& Andre}

\section{DD}

Publicación: Ill. Hort. Pl. 85. 1871.

Colección tipo: Barraquin s.n.

Herbarios:

Nombre común: Desconocido.

Registro departamental: Sin datos.

Regiones Ecológicas: Sin datos; altitud desconocida.

SINANPE: Sin registro.

Herbarios peruanos: Ninguno.

Observaciones: Esta especie no fue reconocida en Brako \& Zarucchi (1993) como endémica. Se conoce solamente del ejemplar tipo, recolectado a mediados del siglo XIX y del que no se tiene información sobre la localidad y hábitat.

\section{Dieffenbachia macrophylla Poepp.}

DD

Publicación: Nov. Gen. Sp. Pl. 3: 90. 1845.

Colección tipo: E.F. Poeppig 1559

Hembarios: W.

Nombre común: D esconocido.

Registro departamental: HU, LO, PA. Regiones Ecológicas: BMHP, BHA; 280 $600 \mathrm{~m}$.

SINANPE: Sin registro.

Hembarios penuanos: USM (1)?.

Observaciones: Hierba conocida originalmente de la cuenca del río Huallaga, recolectada posteriormente de las cuencas de los ríos Pozuzo y Santiago, esta última en el límite entre Amazonas y Loreto. Un ejemplar en el herbario MO proviene de Colombia, pero no ha sido reconfirmado.

\section{Dieffenbachia obliqua Poepp.}

DD

Publicación: Nov. Gen. Sp. Pl. 3: 90. 1845. Colección tipo: E.F. Poeppig s.n.

Herbarios: B.

Nombre común: Desconocido.

Registro departamental: LO.

Regiones Ecológicas: BHA; altitud desconocida.

SINAN PE: Sin registro.

Hembarios peruanos: Ninguno.

Observaciones: Esta especie herbácea no fue listada como endémica en Brako \& Zarucchi (1993). Se conoce solamente de dos localidades en Loreto, una en la cuenca del Marañón y otra, probablemente sobre el Amazonas. Al parecer, no ha vuelto a ser recolectada desde 1924.

\section{Filarum manserichense Nicolson}

$$
\text { EN, Blb(iii)c(iii) }
$$

Publicación: Brittonia 18(4): 349. 1966.

Colección tipo: J.J. Wurdack 2402

Herbarios: BH, F, K, NY, UC, US.

Nombre común: D esconocido.

Registro departamental: AM, CU, LO, SM.

Regiones Ecológicas: $\mathrm{BMHP}, \mathrm{BHA}$; 300-467 m.

SINAN PE: ZRSC

Herbarios peruanos: USM (isotipo+1).

Observaciones: Esta especie es el único representante de este género endémico. Esta hierba tuberosa fue originalmente recolectada de la cuenca del río Santiago y hoy su rango incluye la cuenca del Camisea, en Cusco, y del Huallaga, en San Martín.

\section{Gorgonidium intermedium (Bogner) E.G. Gonç.}

$$
\text { EN, Bla }
$$

Publicación: Aroideana 26: 25. 2003.

Colección tipo: C. Vargas C. 22521

Herbarios: M, US.

Nombre común: Desconocido.

Registro departamental: CU.

Regiones Ecológicas: MA, BPM; 3000 m.

SINAN PE: PNM, SHMP

Henbarios peruanos: Ninguno. 
Observaciones: Esta hierba tuberosa se conoce de unas pocas localidades, en ambientes semixéricos, en el valle del Urubamba. Presenta periodos de latencia, en los cuales no exhibe ni hojas ni inflorescencias; tal vez por ello pasa con frecuencia desapercibida.

\section{Gorgonidium vargasii Bogner \& Nicolson}

$$
\text { VU, B lab(iii) }
$$

Publicación: Bot. Jahrb. Syst. 109(4):

543-547, f. 12- 20. 1988.

Colección tipo: C. Vargas C. 17669

Herbarios: US; CUZ!(2).

Nombre común: Amachu.

Registro departamental: AM, AP, CU, HU, LI, PA.

Regiones Ecológicas: D ST, MA, BMHM; 1050- $3050 \mathrm{~m}$.

SINANPE: Sin registro.

Herbarios penuanos: CUZ (isotipo), USM (1).

Observaciones: Esta hierba tuberosa se conoce de ambientes semixéricos, de valles interandinos y de vertientes orientales. Esta planta permanece la mayor parte del año en estado vegetativo (una sola hoja) o en estado latente. Plantas recolectadas en algunas de las lomas de Lima se atribuyen a esta especie.

\section{Philodendron alatum Poepp.}

\section{VU, Bla}

Publicación: Nov. Gen. Sp. Pl. 3: 87. 1845.

Colección tipo: E.F. Poeppig 1298

Hembarios: W (d).

Nombre común: D esconocido.

Registro departamental: CU, HU, LO, $\mathrm{MD}, \mathrm{SM}$.

Regiones Ecológicas: $\mathrm{BMHP}, \mathrm{BHA}$; 400- $1510 \mathrm{~m}$.

SINANPE: PNM

Herbarios peruanos: Ninguno.

Observaciones: Este bejuco o hierba epífita fue recolectado originalmente en Huánuco en 1829; desafortunadamente, el ejemplar fue destruido durante la Segunda G uerra Mundial (G rayum, 1996). Se conoce de localidades aisladas en las cuencas del Mayo, Huallaga, Alto Madre de Dios, entre otros. Podría encontrarse en la flora boliviana.

\section{Philodendron basivaginatum K. Krause}

$$
\text { CR, B 1ab(iii) }
$$

Publicación: Notizbl. Bot. Gart. BerlinD ahlem 11: 619. 1932.

Colección tipo: E.P. Killip \& A.C. Smith 27424

Herbarios: NY.

Nombre común: D esconocido.

Registro departamental: LO.

Regiones Ecológicas: BHA; $100 \mathrm{~m}$.

SINANPE: Sin registro.

Herbarios peruanos: Ninguno.

Observaciones: Bejuco conocido sólo de la localidad original, en los alrededores de Iquitos. Fue recolectada en 1929, de ambientes boscosos. Por haber sido registrada cerca de esa ciudad, podría ser que esa población no exista, debido a la expansión urbana. No fue incluida en la flórula de Iquitos de Vásquez (1997).

\section{Philodendron densivenium Engl.}

DD

Publicación: Bot. Jahrb. Syst. 37: 132133. 1905.

Colección tipo: A. Weberbauer 1308, A. Weberbauer 3425

Henbarios: $B$.

Nombre común: D esconocido.

Registro departamental: HU, PU.

Regiones Ecológicas: BMHM; 1800$2500 \mathrm{~m}$.

SINAN PE: Sin registro.

Herbarios peruanos: Ninguno.

Observaciones: Este bejuco fue descrito de dos ejemplares recolectados por Augusto Weberbauer en dos localidades diferentes, distantes entre ellas por más de $900 \mathrm{~km}$ en línea recta. Al parecer, no ha vuelto a ser recolectada desde 1903. No se conoce características de su hábitat ni detalles de las localidades originales.

\section{Philodendron devansayeanum L. Linden}

\section{CR, Blab(iii)}

Publicación: Ill. Hort. 42: 376., t. 48. 1895. Colección tipo: Anónimo s.n. Herbarios:

Nombre común: D esconocido.

Registro departamental: PA.

Regiones Ecológicas: BMHM; $2170 \mathrm{~m}$.

SINAN PE: PNYC

Herbarios peruanos: USM (1).

Observaciones: Esta especie es una trepadora, descrita sobre la base de un ejemplar en cultivo en Alemania, en 1894. Una colección reciente en Pasco confirma su presencia en el país.

\section{Philodendron huanucense Engl.}

\section{DD}

Publicación: Bot. Jahrb. Syst. 37: 127. 1905.

Colección tipo: A. Weberbauer 3647

Hembarios: B.

Nombre común: D esconocido.

Registro departamental: $\mathrm{HU}$.

Regiones Ecológicas: BMHP; 600- 700 m.

SINAN PE: Sin registro.

Herbarios peruanos: Ninguno.

Observaciones: Esta hierba se conoce solamente de una localidad en la cuenca del Huallaga. Tiene afinidad con Philodendron tarmense, con la que falta aclarar sus vínculos y su estatus taxonómico.

\section{Philodendron juninense Engl.}

$$
\text { EN, Blab(iii) }
$$

Publicación: Bot. Jahrb. Syst. 37: 126. 1905.

Colección tipo: A. Weberbauer 1823

Herbarios: B; MOL!.

Nombre común: D esconocido.

Registro departamental: HU, JU.

Regiones Ecológicas: BMHP; $1000 \mathrm{~m}$.

SINAN PE: Sin registro.

Hemarios penuanos: MOL (isotipo). 
Observaciones: Esta especie herbácea se conoce de las cuencas de los ríos Huallaga y Perené, ambos valles con paisajes modificados, por la expansión agrícola, desde mediados del siglo XIX. Poco se sabe de sus requerimientos de hábitat y tamaño de las poblaciones.

\section{Philodendron killipii K. Krause}

$$
\text { CR, B1ab(iii) }
$$

Publicación: Notizbl. Bot. Gart. BerlinD ahlem 11: 620. 1932.

Colección tipo: E.P. Killip \& A.C. Smith 24602

Henbarios: B, F, NY, US.

Nombre común: D esconocido.

Registro departamental: JU.

Regiones Ecológicas: BMHM; 1400$1700 \mathrm{~m}$.

SINANPE: Sin registro.

Herbarios peruanos: Ninguno.

Observaciones: Este bejuco de hojas cordadas, se conoce solamente de una localidad en el valle del río Chanchamayo. La localidad original está fuertemente afectada por la deforestación, pues solamente quedan algunos remanentes de sus bosques montanos. Se la considera amenazada por la destrucción del hábitat y por el área de presencia tan limitada.

\section{Philodendron mathewsii Schott}

\section{DD}

Publicación: Bonplandia (Hanover) 7: 29. 1859.

Colección tipo: A. Mathews s.n.

Herbarios: K.

Nombre común: D esconocido.

Registro departamental: HU.

Regiones Ecológicas: BMHP; altitud desconocida.

SINANPE: Sin registro.

Hembarios peruanos: Ninguno.

Observaciones: Esta es una especie trepadora, conocida sólo del material original recolectado a inicios del diglo XIX, probablemente en bosques húmedos premontanos. Poco se conoce de esta especie, tanto sobre el lugar de su procedencia, como de las condiciones de sus poblaciones.

\section{Philodendron membranaceum Poepp.}

\section{CR, Blab(iii)}

Publicación: Nov. Gen. Sp. Pl. 3: 87. 1845. Colección tipo: E.F. Poeppig s.n.

Herbarios: W.

Nombre común: D esconocido.

Registro departamental: HU.

Regiones Ecológicas: BMHP; altitud desconocida.

SINANPE: Sin registro.

Herbarios peruanos: Ninguno.

Observaciones: Este bejuco se conoce sólo de una localidad, en la cuenca media del Huallaga, recolectada en el siglo XIX. Esa parte de la cuenca, está severamente afectada por la deforestación. Probablemente, esta especie está asociada a bosques, pero no hay mayor información sobre su hábitat. No se conoce los vínculos de esta especie con otras en el género.

\section{Philodendron stenophyllum K. Krause}

DD

Publicación: Pflazenr. IV(23D b): 29. 1913.

Colección tipo: A. Weberbauer 3448

Herbarios: B.

Nombre común: D esconocido.

Registro departamental: HU.

Regiones Ecológicas: BMHP; 900- 1000

m.

SINAN PE: Sin registro.

Herbarios peruanos: Ninguno.

Observaciones: Esta especie trepadora se conoce solamente de una localidad, en la cuenca baja del Monzón, la cual ha recibido fuerte impacto ambiental por actividades agrícolas. Forma parte de un complejo de especies que requiere estudio.

\section{Philodendron tarmense Engl.}

DD

Publicación: Bot. Jahrb. Syst. 37: 128. 1905.

Colección tipo: A. Weberbauer 1907

Herbarios: B.

Nombre común: D esconocido.

Registro departamental: JU.

Regiones Ecológicas: BMHP; $1000 \mathrm{~m}$.

SINAN PE: Sin registro.

Herbarios peruanos: Ninguno.

Observaciones: Esta hierba se conoce de una localidad, en el centro del país, en la cuenca del Tulumayo. Tiene afinidad con Philodendron huanuœense, con la que falta aclarar sus vínculos y su estatus taxonómico.

\section{Philodendron weberbauen Engl.}

\section{EN, B lab(iii)}

Publicación: Bot. Jahrb. Syst. 37: 133. 1905.

Colección tipo: A. Weberbauer 1352

Herbarios: $\mathrm{B}$.

Nombre común: D esconocido.

Registro departamental: JU, PU.

Regiones Ecológicas: BMHM; 1500 $1600 \mathrm{~m}$.

SINAN PE: Sin registro.

Herbarios peruanos: Ninguno.

Observaciones: Esta hierba se conoce de dos localidades, aisladas entre ellas por más de $600 \mathrm{~km}$ en línea recta, una en la cuenca del Inambari y otra en la del Tulumayo, ambas en la vertiente oriental. Amenazas potenciales incluyen modificación del hábitat por la deforestación. Al parecer, no ha vuelto a ser recolectada desde 1929.

\section{Spathiphyllum gracilis Bunting}

\section{VU, Blab(iii)}

Publicación: Mem. New York Bot. Gard. 10(3/ 30): 32. 1960

Colección tipo: G. Tessmann 4837

Herbarios: G-DEL, NY.

Nombre común: Patiquina.

Registro departamental: AM, LO.

Regiones Ecológicas: BHA; 120 - 750 m.

SINANPE: Sin registro.

Herbarios peruanos: USM (1). 
Observaciones: Esta hierba fue descrita de una planta recolectada en 1924, de la cuenca del río Santiago, en Amazonas. Podría tratarse de una especie restringida a ambientes arenosos. Ha sido recolectada de localidades aisladas, entre ellas incluyendo los alrededores de Iquitos.

\section{Spathiphyllum tenenum Engl.}

\section{DD}

Publicación: Bot. Jahrb. Syst. 37: 120. 1905.

Colección tipo: E.H.G. Ule 6851

Herbarios: B, G-DEL, MG.

Nombre común: D esconocido.

Registro departamental: SM.

Regiones Ecológicas: BMHP; 1200 m.

SINAN PE: Sin registro.

Herbarios peruanos: Ninguno.

Observaciones: Esta hierba se conoce de los bosques premontanos, de dos localidades en la Amazonía peruana, ambas en la cuenca del Huallaga. Un ejemplar de Colombia podría representar a esta especie, pero falta verificar.

\section{Stenospermation crassifolium Engl.}

$$
\text { EN, B 1ab(iii) }
$$

Publicación: Bot. Jahrb. Syst. 37: 114. 1905.

Colección tipo: A. Weberbauer 3512

Herbarios: $\mathrm{B}$.

Nombre común: D esconocido.

Registro departamental: HU, JU, PA.

Regiones Ecológicas: BMHM; 1400$1900 \mathrm{~m}$.

SINANPE: Sin registro.

Hembarios peruanos: USM (1).

Observaciones: Esta especie herbácea se conoce del centro del país, de localidades aisladas, en las cuencas del Tulumayo, Monzón y Pozuzo. Podría estar representada en el Parque Nacional YanachagaChemillén.

\section{Syngonium gentryanum Croat}

\section{EN, B 1ab(iii)}

Publicación: Ann. Missouri Bot. Gard. 68(4): 612-613, f. 2. 1981.

Colección tipo: A.H. Gentry et al. 18822

Hembarios: MO; USM!.

Nombre común: D esconocido.

Registro departamental: HU.

Regiones Ecológicas: BMHP; 1150$1250 \mathrm{~m}$.

SINAN PE: Sin registro.

Herbarios peruanos: USM (isotipo).

Observaciones: Esta hierba trepadora se conoce de una localidad, entre las cuencas del Aguaytía y Huallaga, en el límite entre Huánuco y Ucayali, y al sur del hoy Parque Nacional Cordillera Azul. Los alrededores de la localidad original, están deteriorados por la deforestación asociada a la expansión agrícola y forestal.
50. Xanthosoma brevispathaceum Engl.

$$
\text { EN, Blab(iii) }
$$

Publicación: Bot. Jahrb. Syst. 37: 137. 1905.

Colección tipo: A. Weberbauer 1821

Herbarios: B; MOL!.

Nombre común: D esconocido.

Registro departamental: HU, JU.

Regiones Ecológicas: BMHP; $1000 \mathrm{~m}$.

SINAN PE: Sin registro.

Herbarios peruanos: MOL (isotipo).

Observaciones: Esta especie herbácea se conoce solamente de dos localidades, en el centro del país, en las cuencas del Pozuzo y Tulumayo, distantes entre ellas por aproximadamente $120 \mathrm{~km}$. No ha vuelto a ser recolectada desde 1929.

\section{Xanthosoma fractum Madison}

\section{DD}

Publicación: Selbyana 5(3-4): 360. 1981. Colección tipo: T. Plowman ex M.T. Madison 6747

Hemarios: F, K, SEL, US.

Nombre común: D esconocido.

Registro departamental: $\mathrm{HU}$.

Regiones Ecológicas: BMHP; altitud desconocida.

SINAN PE: Sin registro.

Herbarios peruanos: Ninguno.

Observaciones: Especie herbácea descrita de una planta cultivada, proveniente de una localidad entre las cuencas del Huallaga y del Aguaytía. Al parecer, no ha vuelto a ser recolectada desde los años 1970. Es poco lo que se conoce sobre su hábitat y poblaciones.

\section{Xanthosoma stenospathum Madison}

EN, Blab(iii)

Publicación: Selbyana 5(3-4): 363. 1981. Colección tipo: T. Plowman ex M.T. Madison 6969

Hembarios: F, K, SEL, US.

Nombre común: D esconocido. Registro departamental: HU, SM. Regiones Ecológicas: BMHP; 500- 1500 m.

SINAN PE: Sin registro.

Herbarios peruanos: Ninguno.

Observaciones: Especie herbácea conocida, al parecer, sólo de dos localidades, ambas en la cuenca del Huallaga. Fue descrita de una planta cultivada, recolectada en los años 1970. 
Mapa del Perú indicando las abreviaturas de los departamentos

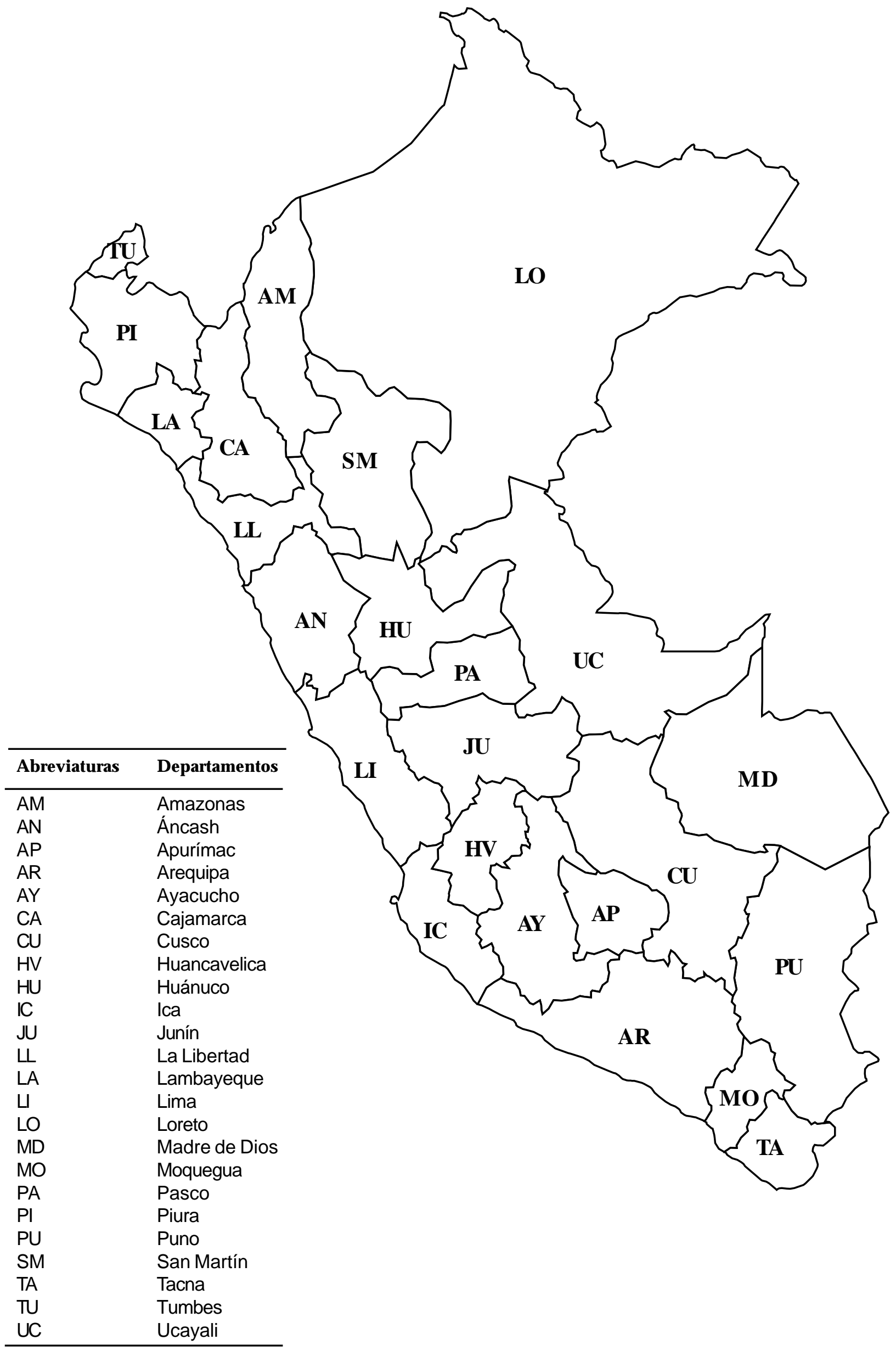

